\title{
Effects of Low-Dose Simvastatin on the Distribution of Plasma Cholesterol and Oxidized Low-Density Lipoprotein in Three Ultra- centrifugally Separated Low-Density Lipoprotein Subfractions: 12- Month, Open-Label Trial
}

\author{
Yasuhiko Homma ${ }^{1}$, Ichiro Michishita ${ }^{2}$, Hiroshi Hayashi ${ }^{3}$, Hiroshi Shigematsu ${ }^{4}$ for Kanagawa Lipid Research \\ Group \\ ${ }^{1}$ Health Evaluation and Promotion Center, Tokai University Hospital, Isehara, Japan \\ ${ }^{2}$ Department of Cardiology, Yokohama Sakae Municipal Hospital, Yokohama, Japan \\ ${ }^{3}$ Department of Internal Medicine, Atami Hospital, International University of Health and Welfare, Atami, Japan \\ ${ }^{4}$ Sato Internal Medical Clinic, Yokohama, Japan
}

\begin{abstract}
Aim: The effects of statins on the distribution of oxidized LDL in plasma LDL subfractions have not been well defined. Effects of 12-month treatment with low-dose simvastatin on the distribution of cholesterol and oxidized LDL in 3 ultracentrifugally separated plasma LDL subfractions were compared in patients with hypercholesterolemia.

Methods: Simvastain was administered to 30 hypercholesterolemic subjects for 12 months at an initial dose of $5 \mathrm{mg} /$ day, which was increased to $20 \mathrm{mg} /$ day via $10 \mathrm{mg} /$ day to decrease plasma LDL- cholesterol (C) lower than $130 \mathrm{mg} / \mathrm{dL}$. Simvastatin dose was fixed after 3 months of treatment. The amounts of cholesterol and oxidized LDL in 3 ultracentrifugally separated plasma LDL subfractions were compared between 0 and 12 months of treatment.

Results: The distribution of ox-LDL skewed to denser LDL fractions, compared with cholesterol in plasma LDL subfractions. Plasma cholesterol in low-density LDL, medium-density LDL and highdensity LDL decreased significantly by $31 \%, 30 \%$, and $25 \%$, respectively $(p<0.0001)$ after 12 months of simvastatin treatment. Plasma oxidized LDL was decreased from $70 \mathrm{U} / \mathrm{L}$ to $56 \mathrm{U} / \mathrm{L}$ in medium-density LDL $(p=0.042)$. Oxidized LDL in low-density LDL and high-density LDL did not change significantly after 12 months of treatment.

Conclusion: Treatment with low-dose simvastatin decreased plasma cholesterol in 3 LDL subfractions and oxidized LDL in medium-density LDL. The decrease of oxidized LDL seemed to be not due to the decrease of cholesterol in plasma LDL subfractions because the decreasing patterns of cholesterol and ox-LDL were different in $3 \mathrm{LDL}$ subfractions.
\end{abstract}

J Atheroscler Thromb, 2010; 17:1049-1053.

Key words; Simvastatin, Plasma lipids, ox-LDL, hs-CRP, LDL subfractions, Plasma lipoprotein subfractions

\section{Introduction}

A statin, simvastatin, has been used for the treatment of hypercholesterolemia and has been proved

Address for correspondence: Yasuhiko Homma, Health Evaluation and Promotion Center, Tokai University Hospital, 143 Shimokasuya, Isehara 259-1193, Japan

E-mail: homma@is.icc.u-tokai.ac.jp

Received: October 25, 2009

Accepted for publication: May 7, 2010 effective for the prevention of coronary artery diseases $(\mathrm{CAD})^{1-3)}$. Low-density lipoprotein (LDL) is a heterogeneous lipoprotein fraction and the atherogenicity of each subfraction is not the same ${ }^{4-6}$. Plasma oxidized $\mathrm{LDL}$ (ox-LDL) is atherogenic ${ }^{7-12)}$, and statins decrease plasma ox-LDL as well as LDL-cholesterol ${ }^{13}{ }^{14}$. Small, dense LDL is easily oxidizable and believed to be very atherogenic ${ }^{15,16)}$, and simvastatin was reported to reduce plasma small, dense $\mathrm{LDL}^{17)}$. We reported the decrease of plasma cholesterol in 2 ultracentrifugally 
separated LDL subfractions after 3 months of treatment with simvastatin ${ }^{18)}$. The decrease in cholesterol in less-dense LDL was more marked than that in dense LDL in that study. The effect of simvastatin on the distribution of plasma ox-LDL levels in LDL subfractions has not been well defined. We investigated the effect of simvastatin on cholesterol and ox-LDL levels in 3 ultracentrifugally separated plasma LDL subfractions after 12 months of treatment with lowdose simvastatin in order to know whether the decreasing patterns of cholesterol and ox-LDL in 3 plasma LDL subfractions are same or not.

\section{Materials and Methods}

\section{Protocol}

The study protocol was approved by the ethics committees of each hospitals and the trial was started after obtaining written informed consent.

Simvastatin was administered to 30 patients with hypercholesterolemia at an initial dose of $5 \mathrm{mg} /$ day after dinner. The duration of the trial was 12 months. The daily dose was increased to $20 \mathrm{mg}$ via $10 \mathrm{mg}$ to reduce plasma LDL-cholesterol lower than $130 \mathrm{mg} /$ $\mathrm{dL}$, and the daily dose was fixed at 3 months of treatment and was not changed until the end of treatment. Plasma lipids, apo(lipo)proteins, ox-LDL, high sensitivity (hs)-CRP, and cholesterol and ox-LDL in $3 \mathrm{LDL}$ subfractions were compared between the start and 12 months of treatment with simvastatin. Blood sampling was made in a 12-hour fasting state.

\section{Laboratory Procedures}

Cholesterol (C) and triglyceride (TG) were measured with an autonalyzer. Apoproteins were estimated with a turbidimetric immunoassay ${ }^{19)}$. Remnant-like particle (RLP)-cholesterol (C) was measured with a precipitation method using monoclonal antibodies against apoprotein (apo) AI and apo B-100 ${ }^{20)}$. Ox-LDL was estimated with an ELISA method using monoclonal antibodies against malondialdehyde (MDA)-LDL and apo $\mathrm{B}^{21}$. One $\mathrm{U} / \mathrm{L}$ of plasma ox-LDL was equivalent to $1 \mathrm{mg} / \mathrm{dL}$ MDA in medium in vitro. Hs-CRP was measured with particle-enhanced immuno- nepherometry ${ }^{22)}$.

Plasma lipoprotein subfractionation with ultracentrifugation into very low-density lipoprotein (VLDL, density; $\mathrm{d}<1.006$ ), intermediate-density lipoprotein (IDL, $1.006<\mathrm{d}<1.019$ ), low-density LDL (ld-LDL, $1.019<\mathrm{d}<1.035$ ), medium-density LDL (md-LDL, $1.035<\mathrm{d}<1.045$ ), high-density LDL (hd-LDL, $1.045<\mathrm{d}<1.063$ ), high-density lipoprotein $(\mathrm{HDL})_{2}(1.063<\mathrm{d}<1.125)$, and $\mathrm{HDL}_{3}(\mathrm{~d}>1.125)$
Table 1. Demographics of subjects

\begin{tabular}{lc}
\hline $\mathrm{N}(\mathrm{M} / \mathrm{F})$ & $30(11 / 19)$ \\
Age $(\mathrm{y} / \mathrm{o})$ & $64.8 \pm 8.4^{1}$ \\
$\mathrm{BMI}\left(\mathrm{kg} / \mathrm{m}^{2}\right)$ & $23.5 \pm 2.7$ \\
Total cholesterol $(\mathrm{TC})(\mathrm{mg} / \mathrm{dL})$ & $264 \pm 34$ \\
Triglyceride $(\mathrm{TG})(\mathrm{mg} / \mathrm{dL})$ & $137 \pm 60$ \\
LDL-C $(\mathrm{mg} / \mathrm{dL})$ & $182 \pm 27$ \\
HDL-C $(\mathrm{mg} / \mathrm{dL})$ & $55 \pm 14$ \\
RLP-C $(\mathrm{mg} / \mathrm{dL})$ & $5.4 \pm 2.5$ \\
Ox-LDL $(\mathrm{U} / \mathrm{L})$ & $162 \pm 56$ \\
Hs-CRP $(\mathrm{ng} / \mathrm{mL})$ & $1,050 \pm 805$ \\
FPG $(\mathrm{mg} / \mathrm{dL})$ & $103 \pm 16$ \\
\hline
\end{tabular}

1. Mean \pm S.D.

was performed sequentially with the method of Havel et al. ${ }^{23)}$. The duration of ultracentrifugation for lipoprotein subfractionation was $16 \mathrm{~h}$ for VLDL, $18 \mathrm{~h}$ for IDL, $20 \mathrm{~h}$ for LDL, and $24 \mathrm{~h}$ for $\mathrm{HDL}_{2}$ at 100,000 $\mathrm{g}$.

\section{Statistical Analysis}

Statistical comparison of serial changes of measured items was performed by ANOVA to identify trends of changes, and further comparison of values between the start and end of the trial was performed with the Dunnett's repeated measurement analysis.

\section{Results}

The demographics of subjects are shown in Table 1. The daily dose of simvastatin at 12 months of treatment was $7.7 \pm 5.2 \mathrm{mg}$ (mean \pm S.D.) $(5 \mathrm{mg}$ for 22 subjects, $10 \mathrm{mg}$ for 4 subjects, and $20 \mathrm{mg}$ for 4 subjects).

Changes of plasma lipids, apoproteins, ox-LDL, and hs-CRP after 12 months of treatment with simvastatin are shown in Table 2. TC, TG, LDL-C, RLP-C. apo B, apo E, and ox-LDL were significantly decreased by 12 months of treatment with simvastatin. Plasma levels of HDL-C, apo AI, and hs-CRP were not changed significantly by treatment with simvastatin.

Distributions of cholesterol and ox-LDL in plasma LDL subfractions at the start of treatment are demonstrated in Fig. 1. The distribution of ox-LDL skewed to higher density fractions, compared with that of cholesterol. A small amount of ox-LDL was recovered in IDL and $\mathrm{HDL}_{2}$ fractions.

Changes in plasma cholesterol and ox-LDL levels in lipoprotein subfractions are shown in Table 3. Plasma levels of VLDL-C, IDL-C, ld-LDL-C, mdLDL-C, hd-LDL-C were significantly decreased after 12 months of treatment with simvastatin. Decreases in 
Table 2. Changes in plasma levels of lipids, apolipoproteins, ox-LDL, and hs-CRP after 12-month treatment with low-dose simvastatin

\begin{tabular}{lcrr}
\hline \multicolumn{1}{c}{ Treatment duration (m) } & 0 & 12 & \multicolumn{1}{c}{$p^{1}$} \\
\hline Total cholesterol (TC) (mg/dL) & $264 \pm 33^{2}$ & $200 \pm 32^{\mathrm{c}}$ & $<0.0001$ \\
Triglyceride (TG) (md/dL) & $137 \pm 60$ & $114 \pm 58^{\mathrm{a}}$ & 0.0415 \\
LDL-cholesterol (LDL-C) (mg/dL) & $182 \pm 26$ & $117 \pm 25^{\mathrm{c}}$ & $<0.0001$ \\
HDL-cholesterol (HDL-C) (mg/dL) & $55 \pm 14$ & $60 \pm 17$ & 0.3825 \\
RLP-cholesterol (RLP-C) (mg/dL) & $6.4 \pm 2.5$ & $3.8 \pm 1.2^{\mathrm{c}}$ & $<0.0001$ \\
Apoprotein AI (apo AI) (mg/dL) & $140 \pm 26$ & $154 \pm 27$ & 0.2566 \\
Apo B (mg/dL) & $143 \pm 19$ & $102 \pm 19^{\mathrm{c}}$ & $<0.0001$ \\
Apo E (mg/dL) & $5.3 \pm 1.4$ & $4.2 \pm 1.2^{\mathrm{c}}$ & 0.0001 \\
Oxidized LDL (ox-LDL) (U/L) & $161 \pm 133$ & $133 \pm 41^{\mathrm{c}}$ & $<0.0001$ \\
Hs-CRP (ng/mL) & $1,040 \pm 818$ & $844 \pm 1,096$ & 0.4502 \\
\hline
\end{tabular}

1. Statistical analysis by ANOVA

2. Mean \pm S.D.

3. Statistical significance by repeated measurement analysis: $\mathrm{a} ; p<0.05$, b; $p<0.01$, c; $p<0.001$

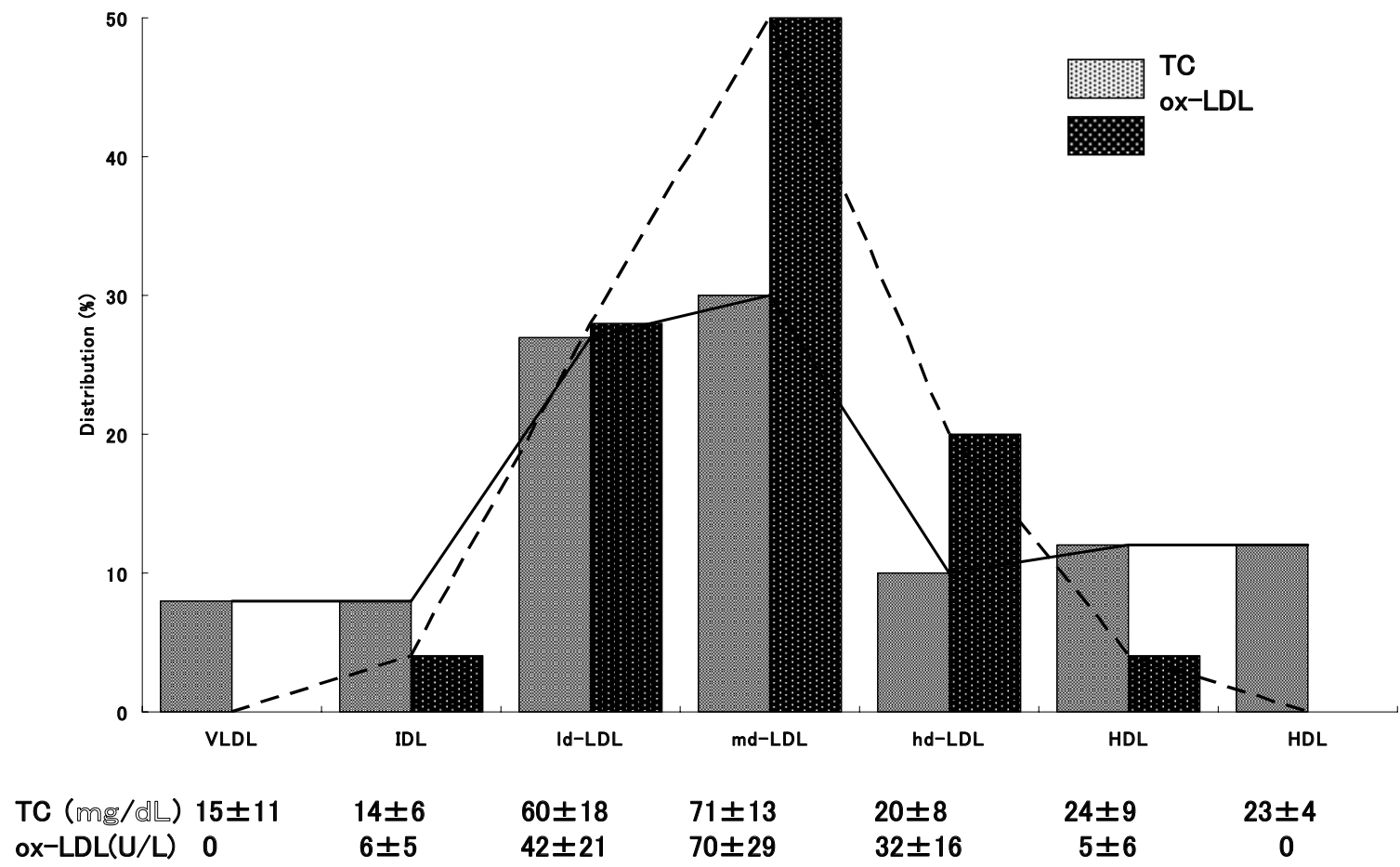

Fig. 1. Distributions of cholesterol and ox-LDL in plasma LDL subfractions at the start of the trial. Absolute amounts are demonstrated at the bottom (mean \pm S.D.).

Small amount of ox-LDL was recovered in IDL and HDL2

the absolute amounts of ld-LDL-C and md-LDL-C were significantly greater than the decrease of hdLDL-C., but percent decreases of cholesterol in $3 \mathrm{LDL}$ subfractions were not significantly different among them (percent decreases of cholesterol: $31 \%$ in ld-LDL, $30 \%$ in md-LDL, and $25 \%$ in hd-LDL). Plasma levels of $\mathrm{HDL}_{2}-\mathrm{C}$ and $\mathrm{HDL}_{3}-\mathrm{C}$ were not changed by simv- astatin treatment. Ox-LDL was significantly decreased only in plasma md-LDL.

\section{Discussion}

Effects of treatment with low-dose simvastatin on the distribution of cholesterol and ox-LDL in 
Table 3. Changes in plasma cholesterol and ox-LDL in lipoprotein subfractions after 12-month treatment with low-dose simvastatin

\begin{tabular}{llrr}
\hline \multicolumn{1}{c}{ Treatment duration (m) } & \multicolumn{1}{c}{12} & $p^{1}$ \\
\hline VLDL-cholesterol (C) (mg/dL) & $15.2 \pm 11.1^{2}$ & $9.9 \pm 8.4^{\mathrm{a}}$ & 0.0235 \\
IDL-C (mg/dL) & $14.0 \pm 5.8$ & $10.1 \pm 4.7^{\mathrm{c}}$ & $<0.0001$ \\
Low-density LDL-C (ld-LDL-C) (mg/dL) & $60.4 \pm 18.3$ & $41.2 \pm 11.6^{\mathrm{c}}$ & $<0.0001$ \\
ld-LDL ox-LDL (U/L) & $41.8 \pm 20.6$ & $35.2 \pm 11.3$ & 0.1795 \\
Medium-density LDL-C (md-LDL-C) (mg/dL) & $70.7 \pm 12.6$ & $49.3 \pm 16.4^{\mathrm{c}}$ & $<0.0001$ \\
md-LDL ox-LDL (U/L) & $70.3 \pm 29.4$ & $56.2 \pm 20.6^{\mathrm{a}}$ & 0.0421 \\
High-density LDL-C (hd-LDL-C) (mg/dL) & $20.3 \pm 7.7$ & $15.3 \pm 5.4^{\mathrm{c}}$ & 0.0002 \\
hd-LDL ox-LDL (U/L) & $32.1 \pm 16.0$ & $31.1 \pm 15.5$ & 0.6572 \\
HDL2-C (mg/dL) & $24.2 \pm 9.0$ & $25.5 \pm 8.4$ & 0.4175 \\
HDL3-C (mg/dL) & $22.6 \pm 3.9$ & $23.5 \pm 3.3$ & 0.6426 \\
\hline
\end{tabular}

1. Statistical analysis by ANOVA

2. Mean \pm S.D.

3. Statistical significance by repeated measurement analysis: a; $p<0.05$, b; $p<0.01$, c; $p<0.001$

plasma LDL subfractions were compared.

Low-dose simvastatin was very effective for the treatment of Japanese subjects with hypercholesterolemia.

Plasma levels of TC, TG, LDL-C, apo B, apo E, and ox-LDL were decreased by treatment with simvastatin, as previously reported $\left.{ }^{1,2}, 18,24,25\right)$; however, plasma levels of HDL-C, apo AI, hs-CRP did not change significantly in this study.

The metabolism of plasma LDL subfractions is complicated. Plasma levels of LDL subfractions are mainly determined by cascade-like catabolism from VLDL to small, dense LDL, direct secretion of LDL subfractions, and their removal via LDL receptors, especially in the liver ${ }^{26-28)}$. The production of VLDL and LDL subfractions is inhibited and their removal via $\mathrm{LDL}$ receptors is stimulated by statin treatment. Plasma levels of VLDL, IDL, ld-LDL, md-LDL, hd-LDL were significantly decreased after-12 month treatment with low-dose simvastatin in this study probably due to these mechanisms. The metabolism of ox-LDL and the effect of simvastatin treatment have not been well defined ${ }^{12)}$. The selective decrease of ox-LDL in md-LDL might be due to the stimulation of minimally oxidized LDL removal through LDL receptors because md-LDL had the highest affinity to LDL receptors among 3 LDL fractions ${ }^{29-32)}$; however, it is impossible to know the mechanisms of changes of cholesterol and ox-LDL in LDL subfractions in this kind of clinical study. The decrease of ox-LDL was speculated to be due to the decrease of cholesterol ${ }^{12)}$. This study suggested that the decrease of ox-LDL was not due to the decrease of cholesterol because the decreasing patterns of cholesterol and ox-LDL in LDL subfractions were different.

We concluded that 12 months of treatment with low-dose simvastatin reduced plasma levels of cholesterol in all 3 LDL subfractions and ox-LDL in md-LDL.

\section{Appendix}

Members of Kanagawa Lipid Research Group: Yasuhiko Homma*, Isehara; Ichiro Michishita, Yokohama; Hiroshi Hayashi, Atami; Hiroshi Shigematsu, Yokohama; Yuichi Fusegawa, Sagamihara; Makoto Ujihara, Yokohama; Toshimasa Fujihara, Narashino; Osamu Mizuno, Ohuna; Hiroshi Yoshida, Kashiwa; Tadashi Yamakawa, Yokohama; Akira Tanaka, Sakato; and Koichiro Homma, Shinjuku

${ }^{*}$ Chief investigator

\section{References}

1) Scandinavian Simvastatin Survival Study Group: Randomized trial of cholesterol lowering in 4444 patients with coronary heart disease: the Scandinavian simvastatin survival study (4S). Lancet, 1994; 344: 1383-1389

2) Matsuzaki M, Kita T, Mabuchi H, et al: Large scale cohot study of the relationship between serum cholesterol concentration and coronary events with low-dose simvastatin therapy in Japanese patients with hypercholesterolemia. Cir J, 2002; 66: 1087-1095

3) Heart Protection Study Collaborative Group: MRC/BHF Heart Protection Study of anti-oxidant vitamin supplementation in 20536 high-risk individuals: a randomized placebo-controlled trial. Lancet, 2002; 360: 7-22

4) Austin MA, Breslow JM, Hennekends CH, Burning JE, Willet WC, Krauss RM: Low-density lipoprotein subclass patterns and the risk of myocardial infarction. JAMA, 
1988; 260: 1917-1921

5) Kwiterovich PO: Clinical relevance of the biochemical, metabolic, and genetic factors that influence low-density lipoprotein heterogeneity. Am J Cardiol, 2002; 90: 30-47

6) Bernis KK, Krauss RM: Metabolic origins and clinical significance of LDL heterogeneity. J Lipid Res, 2002; 43: 1364-1379

7) Salonen JT, Herttuala Y, Yamamoto R, et al: Autoantibody against oxidized LDL and progression of carotid atherosclerosis. Lancet, 1992; 339: 883-887

8) Lehtimaki T, Lehtinen S, Solakivi S, et al: Autoantibody against oxidized low-density lipoprotein in patients with angiographically vertified coronary artery disease. Arterioscl Thromb Vasc Biol, 1999; 19: 23-27

9) Holvoet P, Vanhaaecke J, Janssens S, Werf FV, Collen D: Oxidized LDL and malondialdehyde-modified LDL in patients with acute coronary syndromes and stable coronary artery disease. Circulation, 1998; 98: 1487-1494

10) Ehara S, Ueda M, Naruko T, et al: Elevated levels of oxidized low-density lipoprotein show a positive relation with the severity of acute coronary syndrome. Circulation, 2001; 103: 1955-1960

11) Homma Y: Predictors of atherosclerosis. J Atheroscl Thromb, 2004; 53: 79-88

12) Ishigaki $Y$, Oka Y, Katagiri H: Circulating oxidized LDL: a biomarker and a pathogenic factor. Curr Opinion Lipidol, 2009; 20: 363-369

13) Sasaki S, Kuwahara N, Kunitomo K, et al: Effects of atorvastatin on oxidized low-density lipoprotein, low-density lipoprotein subfraction distribution, and remnant lipoprotein in patients with mixed hyperlipoproteinemia. Am J Cardiol, 2002; 89: 386-389

14) Homma $Y$, Homma $K$, Iizuka S, Iigaya K: Effects of fluvastatin on plasma levels of low-density lipoprotein subfractions, oxidized low-density lipoprotein, and soluble adhesion molecules: a twenty-four-weeks, open-label, dose-increasing study. Curr Therapeutic Res, 2003; 64: 236-247

15) De Graaf J, Hendriks JCM, Demacker PNM, Stalenhoef AFH: Enhanced susceptibility to in vitro oxidation of the dense low-density lipoprotein subfraction in healthy subjects. Arterioscl Thromb Vasc Biol, 1991; 11: 298-306

16) Tribble DL, Holl LG, Wood PD, Krauss RM: Variation in oxidative susceptibility among six low-density lipoprotein subfractions of different density and particle size. Atherosclerosis, 1992; 93: 189-199

17) Bahadir MA, Oguz A, Uzunlulu M, Bahadir O: Effects of different statin treatment on small dense low-density lipoprotein in patients with metabolic syndrome. J Atheroscl Thromb, 2009; 16: 684-690

18) Homma Y, Ozawa H, Kobayashi T, Yamaguchu H, Sakane H, Nakamura H: Effects of simvastatin on plasma lipoprotein subfractions, cholesterol esterification rate, and cholesteryl ester transfer protein in type II hyperlipoproteinemia. Atherosclerosis, 1995; 119: 223-234
19) Noma A, Hata Y, Goto Y: Quantification of serum apolipoprotein A-I, A-II, B, C-II, C-III, and E by the turbidimetric immunoassay: reference values, age- and sexrelated differences. Clin Chim Acta, 1993; 221: 147-157

20) Nakajima K, Saito T, Tamura A, et al: Cholesterol in remnant-like lipoproteins in human serum using monoclonal antiapo B-100 and antiapo A-I immunoaffinity mixed gel. Clin Chim Acta, 1993; 223: 53-71

21) Kotani K, Maekawa M, Kanno T, Kondo A, Toda N, Manabe M: Distribution of immuno-reactive malondialdehyde-modified low-density lipoprotein in human serum. Biochim Biophys Acta, 1994; 1215: 121-125

22) Rifai N, Tracy RP, Ridokoker PM: Clinical efficacy of an automated high-sensitivity $\mathrm{C}$-reactive protein assay. Clin Chem, 1999; 45: 2136-2141

23) Havel RJ, Eder HA, Bragdon JH: The distribution and chemical composition of ultracentrifugally separated lipoproteins in human plasma. J Clin Invest, 1955; 34: 13461353

24) Asztalos BF, Horvath KV, McNamara JR, Roheim OS, Rubinstein JJ, Scaefer EJ: Comparing the effects of five different statins on the HDL subpopulation profiles of coronary heart disease patients. Atherosclerosis, 2002; 164: 361-369

25) Lambertus J, van Tits H, Smilde TJ, et al: Effects of atrovastatin and simvastatin on low-density lipoprotein subfraction profile, low-density lipoprotein oxidizability, and antibodies to oxidized low-density lipoprotein in relation to carotid intima media thickness in familial hypercholesterolemia. J Invest Medicine, 2004; 52: 177-184

26) Shames DM, Havel RJ: De novo production of low density lipoproteins: fact or fancy. J Lipid Res, 1991; 32: 1099-1112

27) Packard CJ, Demant T, Stewart JP, et al: Apoprotein B metabolism and distribution of VLDL and LDL subfractions. J Lipid Res, 2000; 41: 305-317

28) Goldstein JL, Brown MS: The LDL receptor. Arterioscl Thromb Vas Biol, 2009; 29: 431-438

29) Jaakkola O, Solakivi T, Yla-Herttuala S, Nikkari T: Receptor mediated binding and degradation of subfractions of human plasma low-density lipoprotein by cultured fibroblasts. BBA, 1989; 1005: 118-122

30) Nigon F, Lesnik P, Rouis M, Chapman MJ: Discrete subspecies of human low density lipoproteins are heterogeneous in their interaction with the cellular LDL receptor. J Lipid Res, 1991; 32: 1741-1753

31) Campos H, Arnold KS, Balestra ME, Innerarity TL, Krauss RM: Difference in receptor binding of LDL subfractions. Arterioscl Thromb Vasc Biol, 1996; 16: 794-801

32) Yoshida H, Quehenberger O, Kondratenko N, Green S, Steinberg D: Minimally oxidized low-density lipoprotein increases expression of scavenger receptor A, CD36, and macrosialin in resident mouse peritoneal macrophages. Arterioscl Thromb Vas Biol, 1998; 18: 794-802 